\title{
The Ara OB1a association ${ }^{\star}$ Stellar population and star formation history
}

\author{
G. Baume ${ }^{1, \star \star}$, G. Carraro ${ }^{2, \star \star \star}$, F. Comeron ${ }^{3}$, and G. C. de Elía ${ }^{1}$ \\ ${ }^{1}$ Facultad de Ciencias Astronómicas y Geofísicas (UNLP), Instituto de Astrofísica de La Plata (CONICET, UNLP), Paseo del Bosque \\ $\mathrm{s} / \mathrm{n}$, La Plata, Argentina \\ e-mail: [gbaume; gdeelia]@fcaglp. fcaglp.unlp.edu.ar \\ 2 ESO, Alonso de Cordova 3107, Vitacura, Santiago de Chile, Chile \\ e-mail: gcarraro@eso.org \\ 3 ESO, Karl-Schwarzschild-Strasse 285748 Garching bei Munchen, Germany \\ e-mail: fcomeron@eso.org
}

Received 29 July 2010 / Accepted 3 May 2011

\begin{abstract}
Context. The Ara OB1a association is a nearby complex in the fourth Galactic quadrant where a number of young/embedded star clusters are projected close to more evolved, intermediate age clusters. It is also rich in interstellar matter, and contains evidence of the interplay between massive stars and their surrounding medium, such as the rim HII region NGC 6188.

Aims. We provide robust estimates of the fundamental parameters (age and distance) of the two most prominent stellar clusters, NGC 6167 and NGC 6193, that may be used as a basis for studing the star formation history of the region.

Methods. The study is based on a photometric optical survey $\left(U B V I H_{\alpha}\right)$ of NGC 6167 and NGC 6193 and their nearby field, complemented with data from 2MASS-VVV, UCAC3, and IRAC-Spitzer in this region.

Results. We produce a uniform photometric catalogue and estimate more robustly the fundamental parameters of NGC 6167 and NGC 6193, in addition to the IRAS 16375-4854 source. As a consequence, all of them are located at approximately the same distance from the Sun in the Sagittarius-Carina Galactic arm. However, the ages we estimate differ widely: NGC 6167 is found to be an intermediate-age cluster (20-30 Myr), NGC 6193 a very young one (1-5 Myr) with PMS, $\mathrm{H}_{\alpha}$ emitters and class II objects, and the IRAS 16375-4854 source is the youngest of the three containing several YSOs.

Conclusions. These results support a picture in which Ara OB1a is a region where star formation has proceeded for several tens of Myr until the present. The difference in the ages of the different stellar groups can be interpreted as a consequence of a triggered star formation process. In the specific case of NGC 6193, we find evidence of possible non-coeval star formation.
\end{abstract}

Key words. open clusters and associations: general - open clusters and associations: individual: NGC 6193 open clusters and associations: individual: NGC 6167 - stars: formation - stars: pre-main sequence

\section{Introduction}

Ara OB1a has been suggested as an example of triggered star formation in the local Galaxy. A summary of the history of investigations of this region, our present understanding, and possible future studies is presented in Wolk et al. (2008a,b, and references therein). This nearby association contains three open clusters (NGC 6193 in the center of the association, together with NGC 6167 and NGC 6204). In addition, several embedded clusters are present together with both star-forming and quiescent clouds (see Fig. 1 in Wolk et al. 2008a; and Fig. 1 in this paper). The idea that triggered star fromation has taken place in Ara OB1a is suggested by a variety of observations. The prominent cluster NGC 6193 appears to be connected to RCW 108 (=NGC 6188), a rim HII region located westward that marks the edge of a molecular cloud being eroded by the hottest stars in the cluster, where several IRAS sources have been

\footnotetext{
* Two catalogs are only available at the CDS via anonymous ftp to cdsarc.u-strasbg.fr (130.79.128.5) or via http://cdsarc.u-strasbg.fr/viz-bin/qcat?]/A+A/531/A73 $\star \star$ Member of Carrera del Investigador CONICET, Argentina.

$\star \star \star$ On leave from Dipartimento di Astronomia, Università di Padova, Vicolo Osservatorio 2, 35122 Padova, Italy.
}

found (Comerón et al. 2005). Star formation in the molecular cloud may have been triggered by NGC 6193. A connection between the energetic activity of the stars in NGC 6167 and a giant HI bubble surrounding was proposed by Arnal et al. (1987) and Waldhausen et al. (1999, see Fig. 6 at Wolk et al. 2008b).

The ages and distances of the two major clusters NGC 6167 and NGC 6193 derived thus far have been based on the photoelectric and photographic photometry of a few stars, rather than on modern CCD data. Carraro \& Munari (2004) presented CCD data for NGC 6204, but this cluster is located in the outskirts of the association and may not be related to the main star/cluster formation process. Distance estimates to NGC 6193 range from $\sim 1100 \mathrm{pc}$ to $\sim 1400 \mathrm{pc}$, and its age is $\sim 3 \mathrm{Myr}$ (Moffat \& Vogt 1973; Fitzgerald 1987; Kaltcheva \& Georgiev 1992; Vázquez \& Feinstein 1992). As for NGC 6167, the estimated distance ranges from 600 to $1200 \mathrm{pc}$ and the age from $10 \mathrm{Myr}$ to $40 \mathrm{Myr}$ (Moffat \& Vogt 1975; Bruck \& Smyth 1967; Rizzo \& Bajaja 1994).

In the present paper, we present a new CCD-based optical $\left(U B V I_{\mathrm{C}}\right.$ and $\left.\mathrm{H}_{\alpha}\right)$ survey in an attempt to provide more robust determinations of the age and distance of NGC 6193 and NGC 6167. The photometry we present is sufficiently deep to allow us to estimate the nuclear and contraction age, by detecting pre-main sequence (PMS) stars with $\mathrm{H}_{\alpha}$ emission. Therefore 


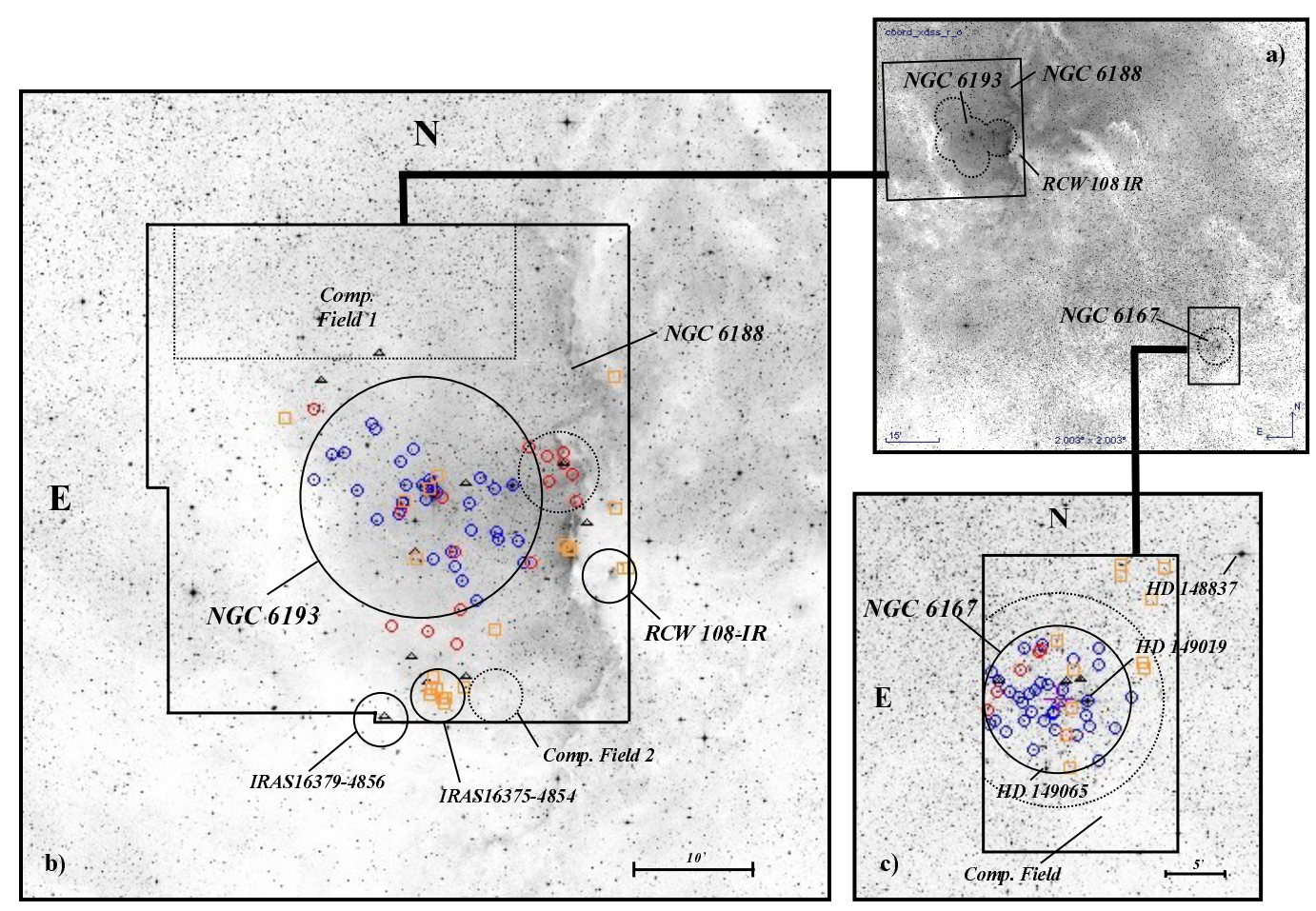

Fig. 1. Second generation Digitized Sky Survey (DSS-2; red filter) images. a) Image centered at $\alpha_{\mathrm{J} 2000}=16: 38: 00 ; \delta_{\mathrm{J} 2000} \sim-49: 15: 20$ and covering a $2^{\circ} \times 2^{\circ}$ field. Solid rectangles centered in NGC 6167 and NGC 6193 indicates areas surveyed with UBVI observations. Dotted circle around NGC 6167 and several dotted circles around NGC 6193 indicates the areas with $\mathrm{H}_{\alpha(\mathrm{on} / \mathrm{off})}$ data. b), c) Detailed images centered respectively at NGC 6193 and NGC 6167 showing main objects in each region and the adopted comparison fields. For the meaning of the symbols see in advance Fig. 4 caption.

we expect to provide firmer estimates of the age and at the same time highlight possible differences between nuclear and contraction ages.

The layout of the paper is as follows. In Sect. 2, we describe the data sources, and their reduction and calibration. In Sect. 3, we present the analysis of the data together with the derivation of the main clusters parameters. In Sect. 4, we derive the main properties of NGC 6167, NGC 6193, and the IRAS 16375-4854 source. Finally, in Sect. 5 we discuss and summarize our results.

\section{Data}

\subsection{Optical data}

Our main data set is based on CCD photometric observations of stars in the region of NGC 6167 and NGC 6193 (see Fig. 1) carried out during several observational runs, and is complemented with available information from the literature. These data include:

- las Campanas Observatory (LCO) $U B V I_{\mathrm{C}}$ images of the region of NGC 6167 acquired during two runs (25 June 2006 and 24 June 2009) using the $1.0 \mathrm{~m}$ Swope telescope equipped with the Site $32048 \times 3150 \mathrm{CCD}$ camera. The field of view (FOV) of these images is about $14 ! 8 \times 22 ! 8$ with a scale 0 .' $^{\prime} 435 /$ pix. The typical $F W H M$ of the data was about $0 .^{\prime} 9$ and airmas values during the observation of the scientific frames were in the range 1.07-1.12;

- Cerro Tololo Inter-American Observatory (CTIO) $U B V I_{\mathrm{C}}$ images of the region covering about $400^{\prime} 0 \times 400^{\prime} 0$ around NGC 6193 acquired during two runs (two frames on 25 and 26 March 2006 and two frames on 21 May 2006). We used the Y4KCAM camera attached to the $1.0 \mathrm{~m}$ telescope, which is operated by the SMARTS consortium ${ }^{1}$. This camera is equipped with an STA $4064 \times 4064 \mathrm{CCD}$ with $15 \mu$ pixels. This set-up provides direct imaging over a FOV $20,0 \times 20.0$ with a scale of $0 ! 289 /$ pix $^{2}$. Typical $F W H M$ of the data was about 0.9 , and airmasses during the observation of the scientific frames were in the range 1.02-1.28;

- complejo Astronómico El Leoncito (CASLEO) $\mathrm{H}_{\alpha-\text { on }}$ $(656.6 \mathrm{~nm})$ and $\mathrm{H}_{\alpha-\text { off }}(666.6 \mathrm{~nm})$ observations. They were performed on 14, 15, 17 and 18 May 2002 in six circular frames (4.5 radius) covering the area of NGC 6167 ( 1 frame) and NGC 6193 (5 frames). We used a nitrogen-cooled detector Tek-1024 CCD and focal reducer attached to the $215-\mathrm{cm}$ telescope (scale 0.'813/pix). Exposure times in $\mathrm{H}_{\alpha-\text { on }}$ and $\mathrm{H}_{\alpha-\text { off }}$ ranged from $2 \mathrm{~s}$ to $150 \mathrm{~s}$;

- other data sources: photometric data for a few bright stars that were found to be saturated in our observations, were taken from Webda database ${ }^{3}$.

Details of all these observations are given in Table 1.

\subsubsection{Reduction}

All frames were pre-processed in a standard way using the IRAF $^{4}$ package CCDRED. For this purpose, zero exposures, and

\footnotetext{
${ }^{1}$ http://www . astro.yale. edu/smarts

2 http://Www. astronomy. ohio-state. edu/Y4KCam/

detector.html

${ }^{3}$ http://www . univie.ac.at/webda

4 IRAF is distributed by NOAO, which is operated by AURA under cooperative agreement with the NSF.
} 
Table 1. Journal of observations of the scientific frames together with the derived photometric calibration coefficients.

\begin{tabular}{|c|c|c|c|c|c|c|}
\hline \multicolumn{7}{|c|}{ Exposure times $[\mathrm{s}]$} \\
\hline Observ. & Frames & $U$ & $B$ & V & $I_{\mathrm{C}}$ & $N$ \\
\hline \multirow[t]{2}{*}{$\mathrm{LCO}$} & long & - & 900 & 600 & 600 & 1 \\
\hline & short & $4 \times 30$ & $2 \times 20$ & 10 & 10 & 1 \\
\hline \multirow[t]{4}{*}{ CTIO } & long & 1500 & 1200 & 900 & 700 & 4 \\
\hline & medium & 200 & 100 & 100 & 100 & 4 \\
\hline & short & 30 & 30 & 30 & 30 & 4 \\
\hline & vshort & 10 & 7 & 5 & 5 & 4 \\
\hline \multicolumn{7}{|c|}{ Calibration and extinction coefficients } \\
\hline Coefficient & \multicolumn{3}{|c|}{$\mathrm{LCO}$} & \multicolumn{3}{|c|}{ CTIO } \\
\hline$u_{1}$ & \multicolumn{3}{|c|}{$+4.528 \pm 0.006$} & \multicolumn{3}{|c|}{$+3.254 \pm 0.007$} \\
\hline$u_{2}$ & \multicolumn{3}{|c|}{$+0.125 \pm 0.011$} & \multicolumn{3}{|c|}{$-0.034 \pm 0.013$} \\
\hline$u_{3}$ & \multicolumn{3}{|c|}{$+0.490 \pm 0.010$} & \multicolumn{3}{|c|}{+0.450} \\
\hline$b_{1}$ & \multicolumn{3}{|c|}{$+2.938 \pm 0.003$} & \multicolumn{3}{|c|}{$+2.015 \pm 0.021$} \\
\hline$b_{2}$ & \multicolumn{3}{|c|}{$+0.050 \pm 0.004$} & \multicolumn{3}{|c|}{$+0.120 \pm 0.025$} \\
\hline$b_{3}$ & \multicolumn{3}{|c|}{$+0.250 \pm 0.010$} & \multicolumn{3}{|c|}{+0.250} \\
\hline$v_{1}$ & \multicolumn{3}{|c|}{$+2.864 \pm 0.003$} & \multicolumn{3}{|c|}{$+1.802 \pm 0.013$} \\
\hline$v_{2}$ & \multicolumn{3}{|c|}{$-0.068 \pm 0.004$} & \multicolumn{3}{|c|}{$-0.048 \pm 0.016$} \\
\hline$v_{3}$ & \multicolumn{3}{|c|}{$+0.160 \pm 0.010$} & \multicolumn{3}{|c|}{+0.160} \\
\hline$i_{1}$ & \multicolumn{3}{|c|}{$+3.757 \pm 0.007$} & \multicolumn{3}{|c|}{$+2.676 \pm 0.020$} \\
\hline$i_{2}$ & \multicolumn{3}{|c|}{$+0.039 \pm 0.009$} & \multicolumn{3}{|c|}{$-0.019 \pm 0.021$} \\
\hline$i_{3}$ & \multicolumn{3}{|c|}{$+0.080 \pm 0.010$} & \multicolumn{3}{|c|}{+0.080} \\
\hline
\end{tabular}

Notes. $N$ indicates the number of fields observed for each cluster region.

sky flats were taken every night. Photometry was performed using IRAF DAOPHOT and PHOTCAL packages. Instrumental magnitudes were obtained using the point spread function (PSF) method (Stetson 1987). Since the FOV is large, a quadratic spatially variable PSF was adopted and its calibration for each image performed using several isolated, spatially well distributed, bright stars (about 25) across each field. The PSF photometry was aperture-corrected for each filter and exposure time. Aperture corrections were computed by performing aperture photometry of a suitable number (about 20) of bright stars in the field. Finally, all data from different filters and exposures were combined and calibrated using DAOMASTER (Stetson 1992).

\subsubsection{Photometric calibration}

Standard stars were selected from particular areas of the catalog of Landolt (1992) (Mark A, PG 1323, and SA 110 for LCO data and SA 101, SA 104, and SA 107 for CTIO data) were used to determine the transformation equations relating our instrumental magnitudes to the standard $U B V I_{\mathrm{C}}$ system. The selection of the fields was made to ensure that the stars had a wide range in colours. Aperture photometry was then carried out for all the standard stars ( $\sim 70$ per night) using the IRAF PHOTCAL package. To tie our observations to the standard system, we use transformation equations of the form:

$$
\begin{array}{lll}
u=U+u_{1}+u_{2}(U-B)+u_{3} X & (\mathrm{rms}=0.04) & (1) \\
b=B+b_{1}+b_{2}(B-V)+b_{3} X & (\mathrm{rms}=0.03) & (2) \\
v=V+v_{1}+v_{2}(B-V)+v_{3} X & (\mathrm{rms}=0.02) & (3) \\
i=I_{\mathrm{C}}+i_{1}+i_{2}\left(V-I_{\mathrm{C}}\right)+i_{3} X & (\mathrm{rms}=0.02)
\end{array}
$$

where $U B V I_{C}$ and $u b v i$ are standard and instrumental magnitudes respectively and $X$ is the airmass of the observation. The used transformation coefficients and extinction coefficients are shown at the bottom of Table 1 .

The $\mathrm{H}_{\alpha-\text { on/off }}$ observations were calibrated by fitting the empirical MS (Didelon 1982; and Feigelson 1983) to the adopted MS stars in each cluster field (see Sect. 3.3 and Fig. 7). In this way, the $\mathrm{H}_{\alpha}$ (on) $-\mathrm{H}_{\alpha}$ (off) index (hereafter $\Delta \mathrm{H}_{\alpha}$ ) has a negative value for objects with a $\mathrm{H}_{\alpha}$ line above the continuum. The relation between the $\Delta \mathrm{H}_{\alpha}$ index and the $\mathrm{H}_{\alpha}$ line width $\left(W_{\mathrm{H} \alpha}\right)$ is given by $\Delta \mathrm{H}_{\alpha}=-2.5 \log \left(1-W_{\mathrm{H} \alpha}[\AA] / 60\right)$.

\subsection{Mid-infrared data}

The NGC 6193 and NGC 6167 regions were observed in the mid-IR by the Infrared Array Camera (IRAC) on-board the Spitzer Space Telescope (SST). IRAC was used to obtain images in four channels $(3.6,4.5,5,8$ and $8.0 \mu \mathrm{m})$ and a catalogue was produced using several of these photometric data points (GLIMPSE $\left.^{5}\right)$. Observations covering NGC 6167 region were included in this catalogue. However, in the case of NGC 6193 and its surrounding area, only IRAC images were available in the SST database belonging to programs P00112, P00191, P03536, P20597, P30570, and P40321.

We performed aperture photometry in all these images by using the IRAF PHOTCAL package following the procedure described by the Spitzer Science Center ${ }^{6}$. Briefly, we run DAOFIND task to look for point sources. We then used the PHOT task using an aperture of 5 pixels and a background annulus from 12 to 20 pixels. Finally, to calibrate our results, we adopted the aperture correction values given in the on-line calibration tables. To avoid false detections, we selected only sources detected in both the four IRAC channels and the $V$ band images.

\subsection{Complementary data and astrometry}

Using the $X-Y$ stellar positions obtained from our data, we correlated their positions with data from the following catalogues: a) "Two-Micron All Sky Survey" (2MASS; Cutri et al. 2003; Skrutskie et al. 2006); b) "Vista Variables in the Vía Láctea" catalogue performed at "Cambridge Astronomy Survey Unit"7 (VVV+CASU; Minniti et al. 2010); and c) "The Third U.S. Naval Observatory CCD Astrograph Catalog" (UCAC3; Zacharias et al. 2010).

Our adopted procedure to perform the astrometric calibration of our data was explained in Baume et al. (2009). The rms of the residuals in the positions were $\sim 0$. $^{\prime} 17$, which is about the astrometric precision of the 2MASS catalogue $\left.(\sim 0)^{\prime} 12\right)$. As for NIR photometry, only stars detected in $V$ filter were selected from NIR catalogues. We adopted 2 MASS values only for the brightest stars $(K<13)$ and VVV + CASU ones for the remainder.

\subsection{Data completeness}

The completeness of the observed star counts is a relevant issue, thus it was computed by means of artificial-star experiments on our data (see Carraro et al. 2005). We created several artificial images by adding at random positions a total of 20000 artificial stars to our true images. These were distributed with a uniform probability distribution of the same colour and luminosity as the real sample. To avoid overcrowding, in each experiment we added the equivalent to only $15 \%$ of the original number of stars. Since in all bands were considered only detections with a $V$ filter counterpart, we performed those experiments on the long exposure image of $V$ filter computing the completeness factor as

\footnotetext{
5 http://www . astro.wisc. edu/glimpse/docs.html

6 http://ssc.spitzer.caltech.edu/irac/

7 http://casu.ast. cam.ac.uk/vistasp/vvv
} 
Table 2. Approximate $V$ magnitud limit values for $80 \%$ and $50 \%$ completeness factors for each studied band.

\begin{tabular}{lcccc}
\hline \hline Filter & \multicolumn{2}{c}{ NGC 6167} & \multicolumn{2}{c}{ NGC 6193} \\
& $80 \%$ & $50 \%$ & $80 \%$ & $50 \%$ \\
\hline$U$ & 13 & 14 & 16 & 17 \\
$B$ & 20 & 21 & 18 & 19 \\
$V$ & 21 & 23 & 21 & 23 \\
$I$ & 21 & 23 & 21 & 23 \\
$\mathrm{H}_{\alpha}$ & 13 & 15 & 13 & 15 \\
$J$ & 16 & 18 & 17 & 19 \\
$H$ & 16 & 18 & 17 & 19 \\
$K$ & 16 & 18 & 17 & 19 \\
{$[3.6]$} & 12 & 15 & 13 & 16 \\
{$[4.5]$} & 12 & 15 & 13 & 16 \\
{$[5.8]$} & 12 & 15 & 13 & 16 \\
{$[8.0]$} & 12 & 15 & 13 & 16 \\
\hline
\end{tabular}

the ratio of the number of artificial stars recovered to the number of artificial stars added. The corresponding factors for the other bands were computed in a relative way as the ratio of the amount of detected stars in each band to the amount of detected stars in $V$ filter corrected by completeness (weighted by diferent area coverage if necessary).

The computed values of the completeness factor for different $V$ magnitude bins are listed in Table 2. We note that for NGC 6167, only short exposures were obtained in the $U$ filter, which explains the poor completeness of its catalogue in this band. Notwithstanding the completeness is sufficient to ensure a reliable estimate of the cluster parameters.

\subsection{Final catalogues}

The above procedure allowed us to build two astrometric, photometric (13 bands) catalogues covering the regions of both NGC 6167 (33193 objects) and NGC 6193 (39338 objects). These two catalogues constitute the main observational database used in this study. A solid analysis of the behavior of the stellar energy distributions (SEDs) can be carried out with this tool, thus preventing possible degeneracies in the photometric diagrams and allowing us to obtain more reliable results. Both catalogues are available only in electronic form at the Centre de Donnes astronomiques de Strasbourg (CDS). They includes $X-Y$ positions, 2MASS identification (when available), equatorial coordinates (epoch 2000.0), and optical (UBVI and $\left.\mathrm{H}_{\alpha-\text { on/off }}\right)$, NIR 2MASS/VVV (JHK), and MIR IRAC-SST (3.6, $4.5,5,8$ and $8.0 \mu \mathrm{m})$ photometry.

\subsection{Clusters centers and sizes}

Radial stellar density profiles provide an objective tool to determine the size of a star cluster. They were computed, in a first attempt, starting from the estimated position of the cluster centers obtained by visual inspection of the second generation Digitized Sky Survey (DSS-2, red) plates, using our brightest catalogued stars ( $V<16-17$ depending on the cluster) and all the brightest 2MASS catalogued objects $(K<13-13.5$ depending on the cluster). We then repeated the procedure several times, slightly changing in each case the position of the centers and using different magnitude bins. We finally adopted these cases that produce the most uniform fall off in both bands. According to the variation observed in different experiments, we estimated that the clusters centers have $\sim 0.5$ uncertainty in each coordinate.
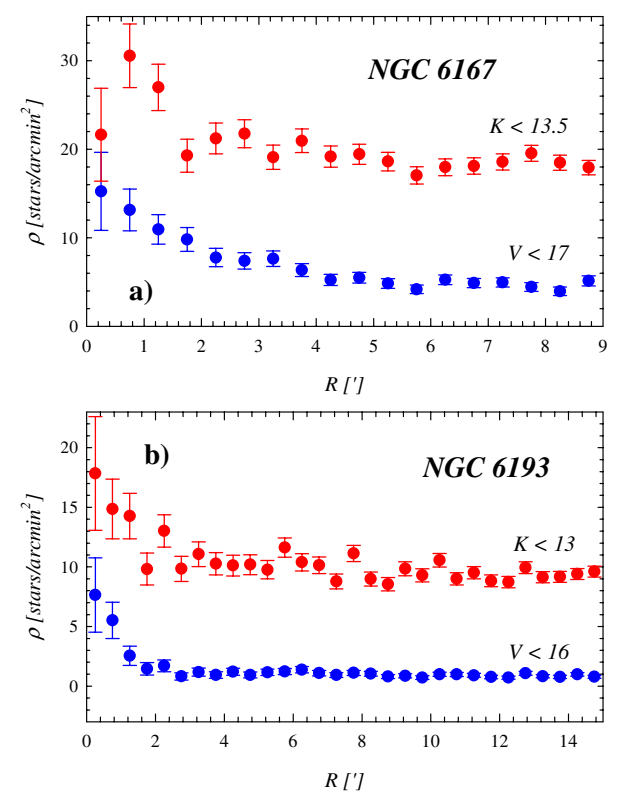

Fig. 2. Radial density profiles for NGC 6167 (upper panel) and NGC 6193 (lower panel). Lower plots (blue) correspond to CCD data $(V<16)$, whereas upper plots (red) correspond to 2MASS data $(K<$ 13). Poisson error bars $(1 \sigma)$ are also shown.

The cluster radial density profiles were computed in the usual way (see Baume et al. 2004), namely calculating the surface density in concentric, equal-area rings. Since we applied this method to both the optical ( $V$ filter) and the 2MASS infrared data ( $K$ filter), this allowed us to detect the possible influence of interstellar material. In both clusters, noticeable differences between the profiles for each band are detected. As a result, the sizes were estimated taking into account mainly the $K$ filter, where absorption effects are minimized.

The adopted centers and radii are indicated in Table 4 and Fig. 1. Centers are close to the ones given by Dias et al. (2002) or by the SIMBAD database, although the radius values are larger than those compiled by Dias et al. (2002), which are based only on visual inspection. The radial density profiles are shown in Fig. 2. Our data completely cover the clusters and sample also a significant portion of the surrounding field. This allows us to select different zones in each region and study them separately to understand the behavior of the stellar population in the directions of the clusters and their neighborhood (see Fig. 1).

\section{Data analysis}

\subsection{Proper motion analysis}

To obtain additional information to confirm and support the membership assignment, we used kinematic data provided by UCAC3 and Hipparcos catalogues (the later one was necessary for few bright stars with unacceptable large errors in UCAC3). Therefore, we used a sample of stars with UCACmag $<14$ and proper motion errors smaller than 10 mas/yr within the radius adopted for NGC 6167 and NGC 6193. To identify the members of the clusters we adopted the method developed by Cabrero-Caño \& Alfaro (1985). This statistical algorithm fits the observed proper-motion distribution with the sum of two Gaussian distributions, one elliptical for the field and one circular for the cluster. Moreover, we took into account the observed errors in the proper motions for individual stars, which was suggested by Zhao \& He (1990). The parameters of the 

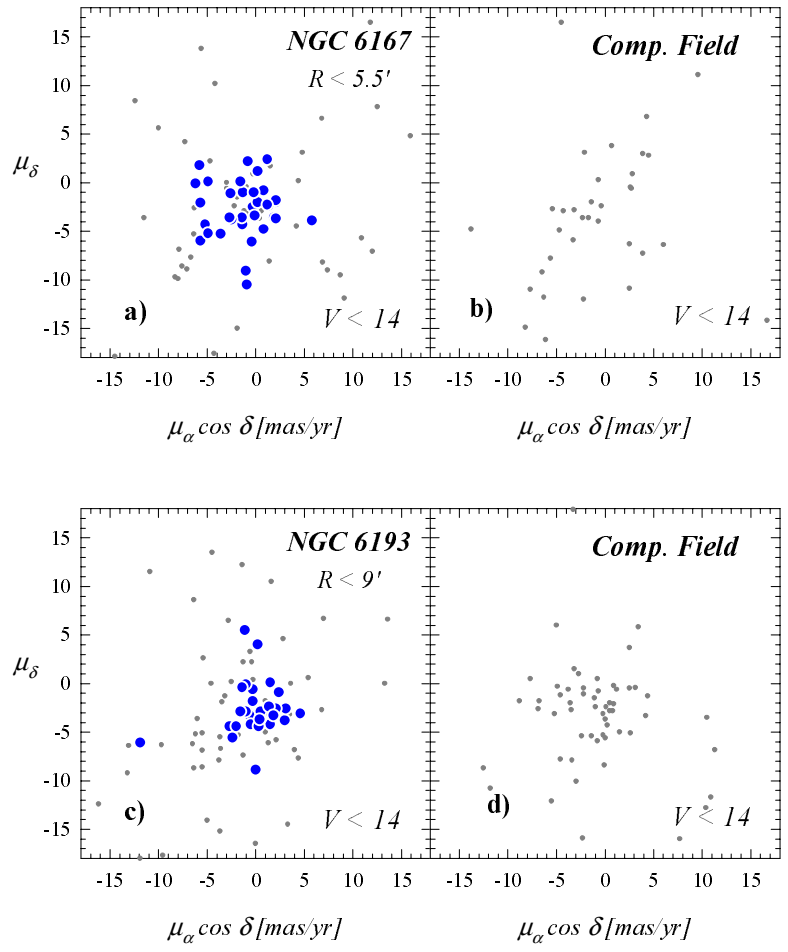

Fig. 3. Proper motion VPDs for adopted cluster areas of NGC 6167 and NGC 6193 and their corresponding comparison fields. Filled (blue) circles are adopted as likely members for each cluster.

Table 3. Kinematic results from brightest stars $(V<14)$ in NGC 6167 and NGC 6193 regions.

\begin{tabular}{cccc}
\hline \hline & Parameters & NGC 6167 & NGC 6193 \\
\hline \multirow{3}{*}{ Cluster } & $\mu_{\alpha \cos \delta_{\mathrm{c}}}$ & -0.85 & 0.54 \\
& $\mu_{\delta_{\mathrm{c}}}$ & -2.61 & -2.91 \\
& $\sigma$ & 1.40 & 1.57 \\
\hline \multirow{4}{*}{ Field } & $\mu_{\alpha \cos \delta_{\mathrm{f}}}$ & -3.72 & -3.66 \\
& $\mu_{\delta_{f}}$ & -3.85 & -6.16 \\
& $\Sigma_{1}$ & 8.34 & 6.76 \\
& $\Sigma_{2}$ & 8.03 & 9.12 \\
& $\rho$ & 0.24 & 0.28 \\
\hline
\end{tabular}

Notes. $\left(\mu_{\alpha \cos \delta_{\mathrm{c}}}, \mu_{\delta_{\mathrm{c}}}\right)$ and $\left(\mu_{\alpha \cos \delta_{\mathrm{f}}}, \mu_{\delta_{\mathrm{f}}}\right)$ are the cluster and field proper motion centers in mas/yr; $\sigma$ and $\left(\Sigma_{1}, \Sigma_{2}\right)$ are the cluster and field proper motion dispersions in mas/yr; $\rho$ is the obtained correlation coefficient.

two Gaussian distributions could then be determined from the maximum-likelihood technique. A summary of the application of these methods can be found in Fernández Lajús et al. (in prep.). We note that the present study differs from that developed by Dias et al. (2006) who used a total sample of 416 stars extracted from the UCAC2 catalogue for the clusters under consideration.

Table 3 shows the results obtained for NGC 6167 and NGC 6193. Finally, Fig. 3 presents the vector point diagram (VPD) for each cluster region. The adopted members (see Sect. 3.2) produce a clear clump separated from the field stars.

\subsection{Optical photometric diagrams}

To perform the final membership assignment of the stars to the clusters, we analyze both: a) the individual stellar positions in all the photometric diagrams (e.g. Baume et al. 2004, 2006, 2009); and b) the obtained membership probabilities values computed from the kinematic information (see Sect. 3.1). In the case of NGC 6193, $\mathrm{H}_{\alpha \text { (on/off) }}$ data were also considered (see Sect. 3.3). The optical two-colour diagrams (TCDs) and colour-magnitude Diagrams (CMDs) of NGC 6167 and NGC 6193 are shown in Figs. 4 and 5.

Individual positions of all stars down to $V=14$ were simultaneously examined in all the diagrams. At fainter magnitudes, UCAC3 does not provide reliable solutions. This makes contamination by field stars more severe, preventing an easy identification of faint cluster members using this method.

The above selection of stars for both clusters allowed us to fit the blue edge of the adopted members with a properly reddened Schmidt-Kaler (1982) zero-age main-sequence (ZAMS) in the $U-B$ versus (vs.) $B-V$ diagrams. We adopted the wellknown relation $E_{U-B} / E_{B-V}=0.72+0.05 E_{B-V}$ and obtained the corresponding $E_{B-V}$ value for each cluster. The $B-V$ vs. $V-I$ diagram is routinely used as a diagnostic to detect deviations from the normal reddening law in a given direction. In this case the cluster diagrams suggest a normal law $\left(R=A_{V} / E_{B-V}\right)$ for NGC 6167 and a marginally different behavior for NGC 6193 confirming previous results of Wolk et al. (2008b).

We then simultaneously fitted a ZAMS or main sequence (MS) to all the CMDs to obtain cluster distance moduli, adopting $R=3.1$ for NGC 6167 and $R=3.2$ for NGC 6193. Finally, in Fig. 6a we compared the observed upper MS of NGC 6193 with theoretical isochrones for solar metallicity, mass loss, and overshooting (Marigo et al. 2008). Some scatter (likely caused by the presence of binaries and rapid rotators) is present in our data and this prevents a unique isochrone solution. However, it is still possible to obtain an estimate of the nuclear age. All the resulting parameters are summarized in Table 4.

\section{3. $H_{\alpha}$ photometric diagrams}

One of the aims of this work was to detect stars with $\mathrm{H}_{\alpha}$ emission. For this purpose we used the $\Delta \mathrm{H}_{\alpha}$ index described in Sect. 2.1.2. Similar indices were used in previous works (Adams et al. 1983; Sung et al. 1998; Baume et al. 2003).

Figure 7 presents the $V$ vs. $\Delta \mathrm{H}_{\alpha}$ diagram for all stars with measured $\Delta \mathrm{H}_{\alpha}$ index together with the corresponding ZAMS in this plane. Most stars follow approximately the ZAMS location indicating that they have no significant $\mathrm{H}_{\alpha}$ emission allowing us to perform the calibration mentioned in Sect. 2.1.2. Therefore positive $\Delta \mathrm{H}_{\alpha}$ index values correspond to absorption features and negative values to emission ones.

To detect stars with, on average, high $\mathrm{H}_{\alpha}$ emission, we identified likely $\mathrm{H}_{\alpha}$ emission objects with the brighest stars $(V<15.5)$ that had $\Delta \mathrm{H}_{\alpha}-3 e_{\Delta \mathrm{H} \alpha}$ negative values.

As for the distribution of $\mathrm{H}_{\alpha}$ emitters, we note in Fig. 1 an evident concentration about 7.'0 westward of NGC 6193. This concentration has a diameter $\sim 2^{\prime}$ and is located close to the NGC 6188 rim $\left(\alpha_{\text {J2000 }} \sim 16: 40: 30 ; \delta_{\text {J2000 }} \sim-48: 44: 20\right.$; see Fig. 1b), just at the edge of the SFO 80 cloud (Sugitani et al. 1991; Sugitani \& Ogura 1994). This group of $\mathrm{H}_{\alpha}$ emitters closely corresponds to the MSX emission peaks at $8 \mu \mathrm{m}$ found inside the cloud by Urquhart et al. (2009). All these elements seems to show different phases of stellar formation processes that are currently on-going. A particular spatial coincidence between a class II object (see Sect. 3.4) and an $\mathrm{H}_{\alpha}$ emitter was found very near to GSC 08329-03510. This star is placed just at the border of the HII region NGC 6188 and very close to the center of the concentration of $\mathrm{H}_{\alpha}$ emitters (see Sect. 3.3). 

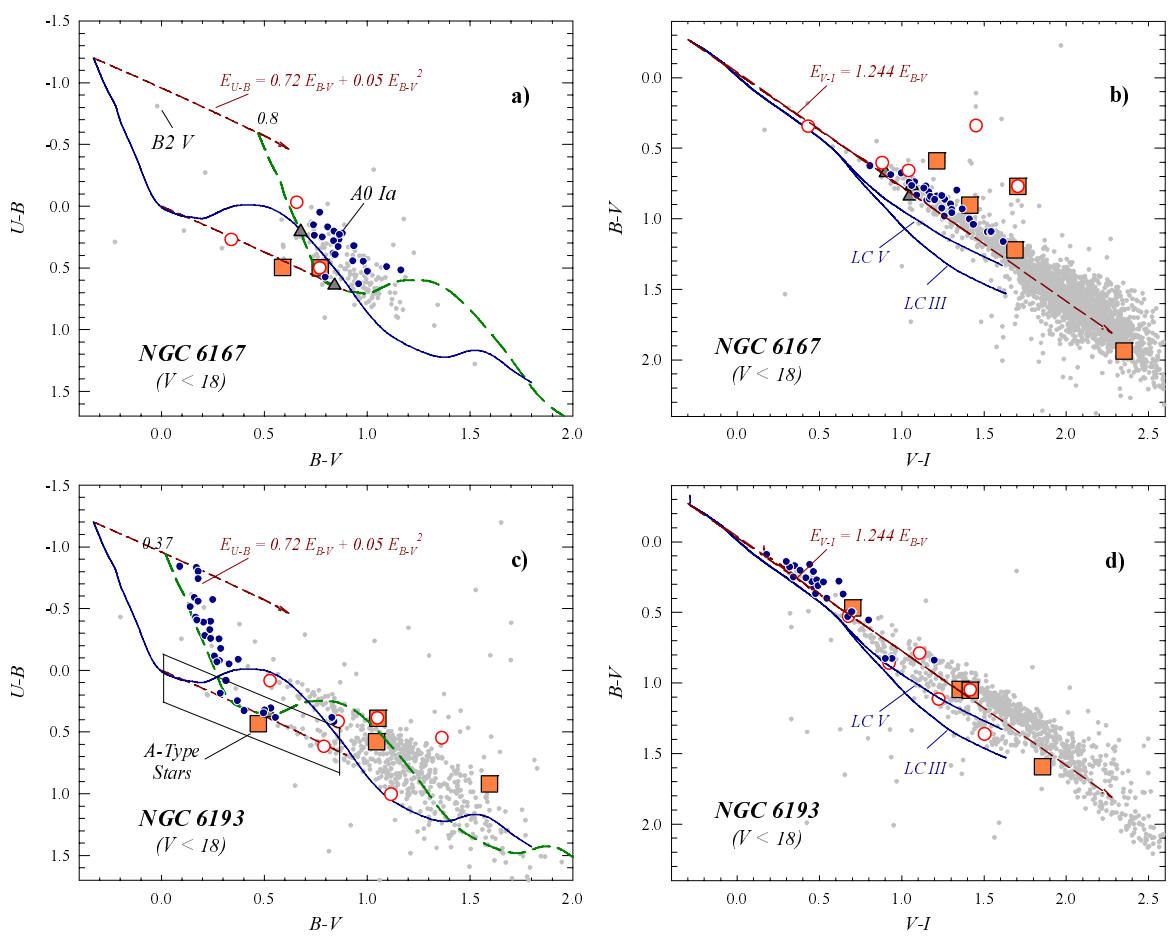

Fig. 4. Optical TCDs of stars located in NGC 6167 (upper panel) and NGC 6193 (lower panel) regions. $U-$ $B$ vs. $B-V$ diagrams: filled (blue) circles are adopted likely members for each cluster, open (red) circles are likely $\mathrm{H}_{\alpha}$ emitter stars; big (orange) squares and filled (grey) triangles are respectively considered probable class I and class II objects (see Sect. 3.4 and also the comments for each cluster); light dots are considered as field stars. The solid (blue) curve is the SchmidtKaler (1982) ZAMS, while dashed (green) lines are the same ZAMS, but shifted along the reddening line (red) by the adopted colour excesses indicated above them. They correspond to the adopted values for the cluster stars (see Table 4). $B-V$ vs. $V-I$ diagrams: symbols have the same meaning as in plots a) and c), whereas solid curves (blue) are intrinsic colours for luminosity class V and III from Cousins (1978a,b). Dashed (red) arrows indicate the normal reddening path $\left(R_{V}=3.1\right)$.
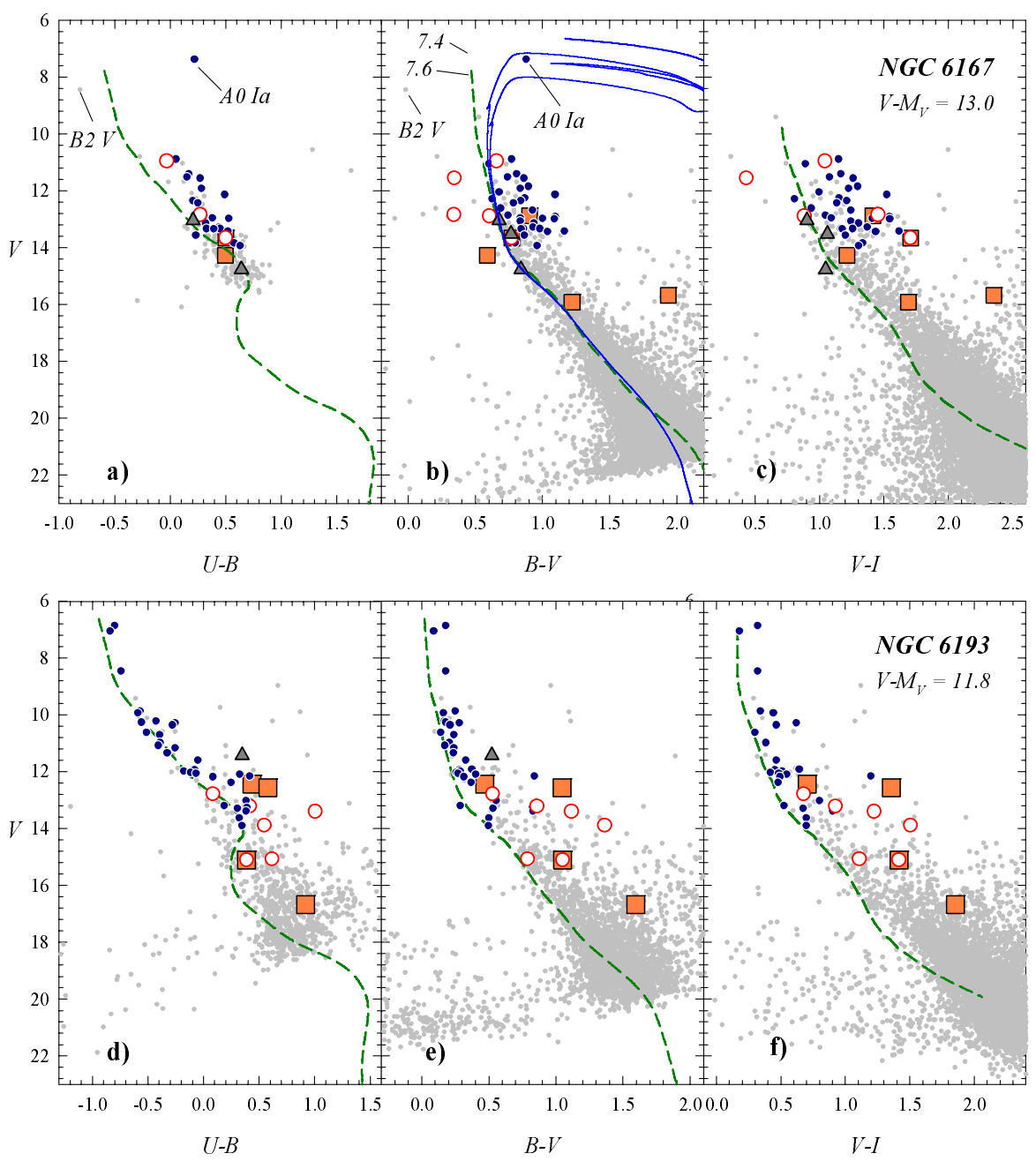

Fig. 5. Optical CMDs of stars located in NGC 6167 (upper panels) and NGC 6193 (lower panels) regions. Symbols are the same as in Fig. 4. Dashed (green) curves are the Schmidt-Kaler (1982) empirical ZAMS and the MS path from Cousins (1978a,b). Solid curves are Marigo et al. (2008) isochones. All the reference curves are corrected by the adopted colour excess and apparent distance modulus for each cluster (see Table 4). 

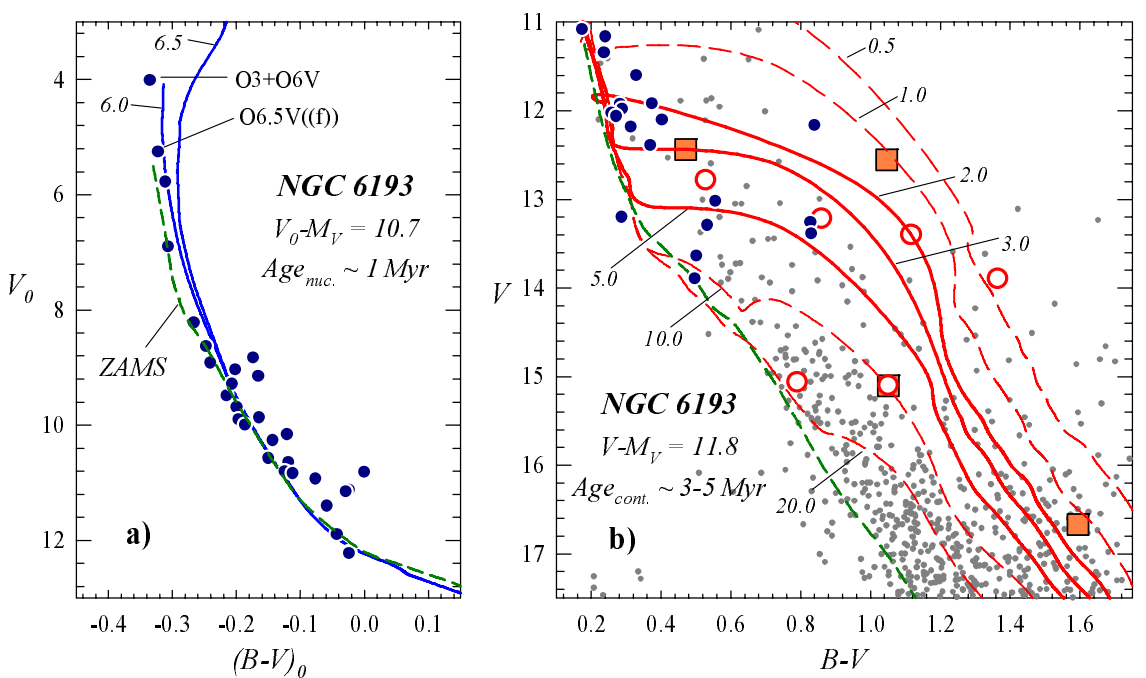

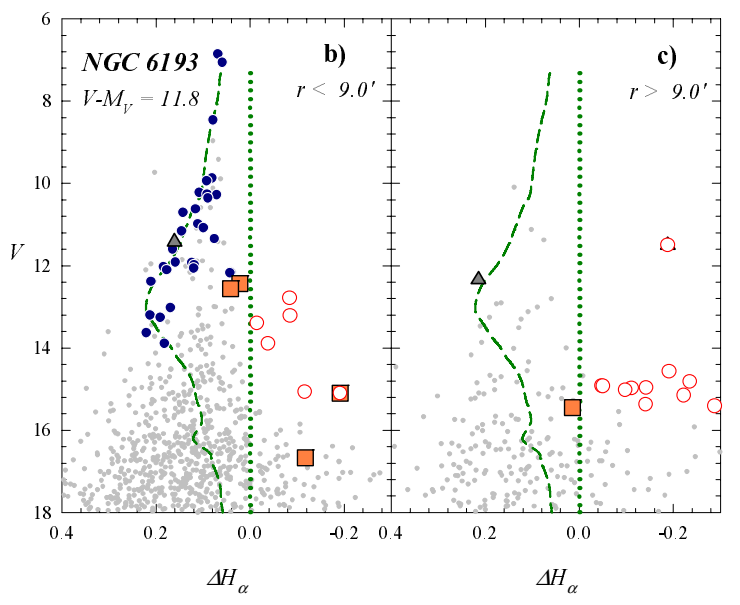

Fig. 6. Detailed CMD of stars located in NGC 6193 region. Symbols are the same as in Fig. 4. Dashed (green) curves are the Schmidt-Kaler (1982) empirical ZAMS; solid (blue and red) curves are Marigo et al. (2008) and Siess et al. (2000) isochones for $z=0.02$. All the reference curves are corrected by the adopted colour excess value and apparent distance modulus (see Table 4).

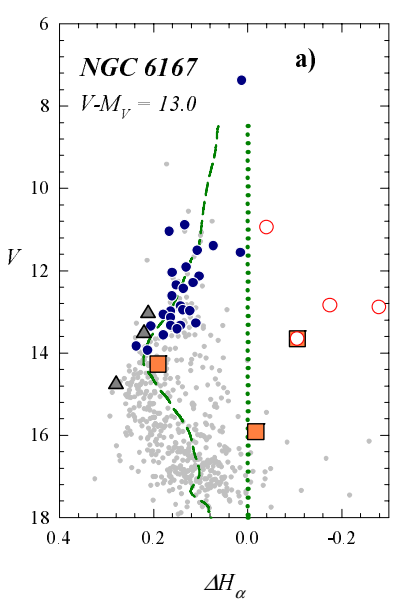

\subsection{Infrared photometric diagrams}

Contracting stars emit an excess of radiation in the IR relative to their photospheric emission. This IR excess is considered to be produced by thermal emission from the circumstellar material. Therefore, this kind of objects are grouped into different "classes" according to their SEDs (Lada 1987; Andre et al. 1993). Thus, NIR and MIR photometric observations allow one to detect and distinguish this kind of sources.

We adopted the selection scheme presented in Gutermuth et al. (2008). This method is based on the location of the observed objects in the $(K-[3.6])_{0}$ vs. $([3.6]-[4.5])_{0}$ plane using the standard dwarf star colours from Bessell \& Brett (1988) and Flaherty et al. (2007) colour excess ratios to obtain de-reddened colours. We then confirmed the selection by examining the locations in the $J-H$ vs. $H-K$ diagram (see Fig. 8).

This analysis allowed us to recognize class I and class II/III candidates among our data. Their spatial distribution (see Fig. 1b) reveals that they are associated with IRAS sources and/or with denser molecular clouds reinforcing the hypothesis that they are indeed YSOs.

\section{Intrinsic cluster properties}

\subsection{NGC 6167}

NGC 6167 appears to be an intermediate-age cluster with significant differential reddening across its surface (see its TCDs and
CMDs). This is consistent with the previous polarimetric results found by Waldhausen et al. (1999).

The age of the cluster was computed by fitting the distribution of the stars in $U-B$ and $B-V$ CMDs with isochrones. We paid particular attention to the brightest star in the CMDs, which is HD $149019=$ CPD-49 9414 (A0 Ia star from SIMBAD). It has a high membership probability value from the kinematic analysis (see Sect. 3.1), and is placed near the cluster center (see Fig. 1c). The spectrophotometric method also infers for this star a distance moduli of $\left(V_{0}-M_{V}\right)_{\mathrm{sp}}=10.5$, which is consistent with the adopted value for the cluster. As a consequence, this star is adopted as a likely cluster member and the best-fit isochrone is that for 20-30 Myr. In addition, only few stars in the field of this cluster exhibit a sizeable $\mathrm{H}_{\alpha}$ emission (see Fig. 7a), bringing very support to this age value.

The second bright star in the cluster region is HD $149065=$ CPD-49 9421 (B2 V star from SIMBAD). This star seems to be a foreground star according to its location in the photometric diagrams. In the $U-B$ vs. $B-V$ diagram, in particular, it shows a colour excess smaller than the cluster stars (0.24 and 0.80 , respectively). Its spectrophotometric distance moduli is $\left(V_{0}-M_{V}\right)_{\mathrm{sp}}=10.1$, which is significantly smaller than the one adopted for NGC 6167 (10.5). These differences might be caused by the Galactic absorption in the cluster direction (see Neckel \& Klare 1980), which is systematically greater by $\sim 2$ mag (decreasing the mean brightness from less than 1 mag to almost $3 \mathrm{mag}$ ) at $\sim 1 \mathrm{kpc}$ from the Sun. On the other hand, 

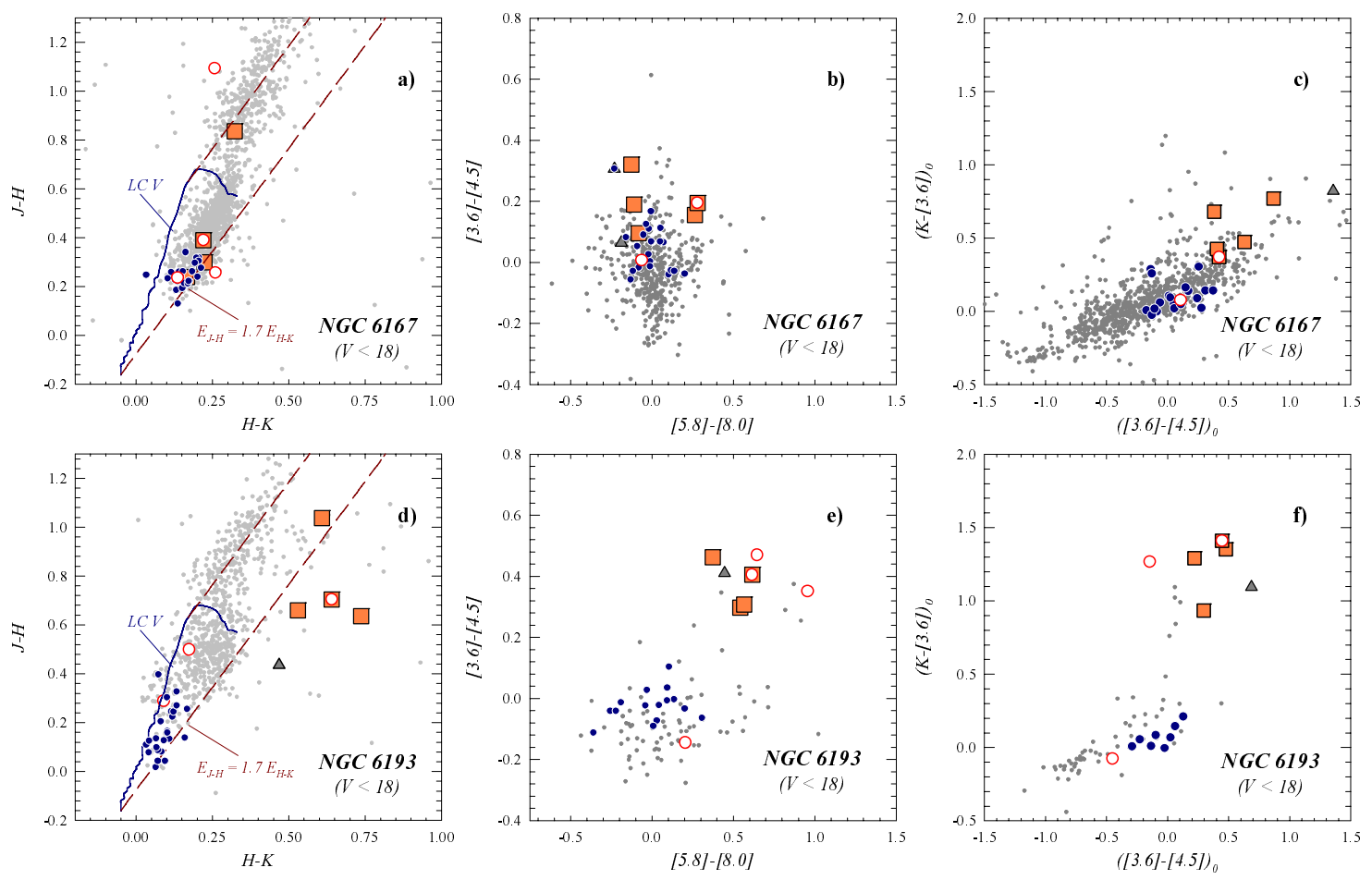

Fig. 8. Infrared CCDs of stars located in NGC 6167 (upper panels) and NGC 6193 (lower panels) regions. Symbols are the same as in Fig. 4. Solid curves are the intrinsic Koornneef (1983) colours for MS stars.

HD 149065 is almost at the border of the adopted cluster region (see Fig. 1c), so we can assume that it is probably an interloper.

With an age of 20-30 Myr, it is unlikely that the sources considered as class I/II objects in Fig. 8 are real YSOs. These objects appear to be young based only on the MIR diagram (Fig. 8c), although their location in the NIR diagram (Fig. 8a) should be different to adopt them as YSOs.

\subsection{NGC 6193}

This cluster looks very young. Figure 6 a presents the reddeningcorrected CMD obtained for the adopted likely cluster members in the upper MS. Differential reddening was removed using individual colour excesses from Fig. $4 \mathrm{c}$ and adopting $R=3.2$. The isochrone fitting method (see Sect. 3.2) then provides the nuclear age ( $\sim 1 \mathrm{Myr})$. This result is compatible with the latest spectroscopic analysis of its brightest cluster member (star HD 150136; O3+O6V, Niemela \& Gamen 2005).

The lower MS of NGC 6193 shows quite a significant $M_{V}$ scatter at any colour. There are also several objects in the cluster field, which were those identified as $\mathrm{H}_{\alpha}$ emitters and a few as class II objects (see Sect. 3.4). Figure 6b presents the location of these objects in a un-corrected CMD. These objects are all located above ( 1-2 mag) the cluster ZAMS. Both effects, spread and location above the ZAMS, strengthen the idea that we are in the presence of a young cluster (cf. Fig. 3 in Preibisch \& Zinnecker 1999). The observed spread might have intrinsic causes such as binarity/multiplicity, the random distribution of the orientation of the accretion discs around single and multiple systems, as well as external causes such as differential reddening, field star contamination, and photometric errors.

The lower MS shape allowed us to derive an independent estimate of the cluster age (the contraction age) by comparing it with theoretical PMS isochrones. We achieved this by adopting the models computed by Siess et al. (2000). We note that it was

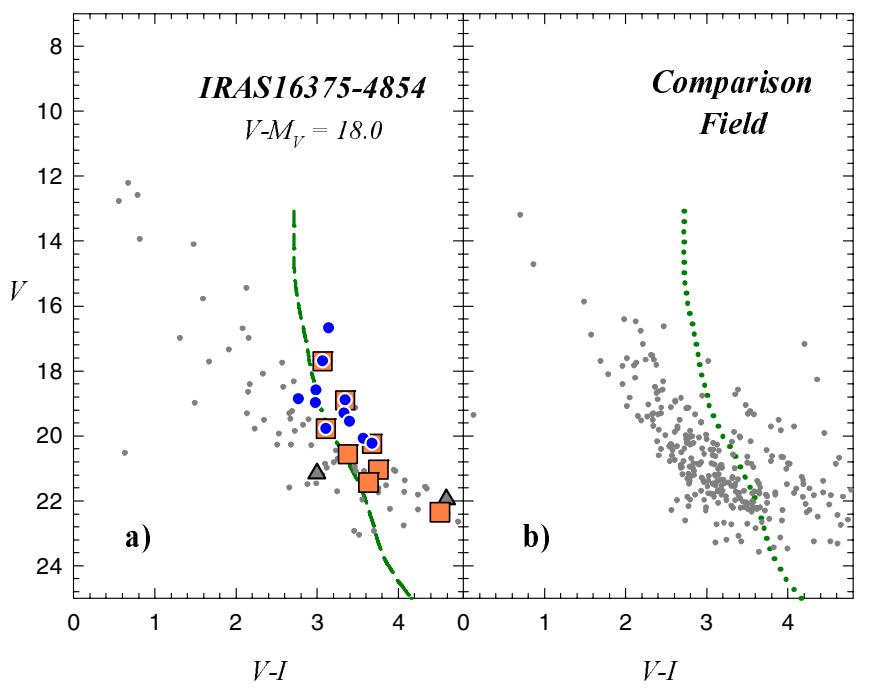

Fig. 9. $V$ vs. $V-I$ diagrams of stars located in IRAS 16375-4854 region and its corresponding comparison field. Filled (blue) circles are adopted members for each clump. Curves have the same meaning as in Fig. 4.

impossible to find a unique isochrone that fitted the entire lower MS, but that we had to use a family of isochrone encompassing the stars loci in the CMD. Despite this, the mean location of the lower MS (see Baume et al. 2003) could be fitted with a group of isochrones that correspond to an age value marginally older than the nuclear age ( $\sim 3-5 \mathrm{Myr})$.

We are aware that the accuracy of the contraction age might only be considered as an order of magnitude. This is because the large dispersion of objects in the lower MS may also reflect a non-coeval process of stellar formation. This is reinforced by a) the presence of objects of different nature in that area of the CMD such as stars near the ZAMS, $\mathrm{H}_{\alpha}$ emitters, and class II 
Table 4. Parameters of the analyzed stellar groups.

\begin{tabular}{|c|c|c|c|c|c|c|c|c|c|c|}
\hline \multirow[t]{2}{*}{ Stellar group } & \multicolumn{2}{|c|}{ Center } & \multirow{2}{*}{$\begin{array}{c}\text { Radius } \\
{\left[{ }^{\prime}\right]}\end{array}$} & \multirow[t]{2}{*}{$E_{B-V}$} & \multirow[t]{2}{*}{$R$} & \multirow[t]{2}{*}{$V-M_{V}$} & \multirow[t]{2}{*}{$A_{V}$} & \multirow[t]{2}{*}{$V_{0}-M_{V}$} & \multicolumn{2}{|c|}{ Age [Myr] } \\
\hline & $\alpha_{2000}$ & $\delta_{2000}$ & & & & & & & Nuclear & Contrac. \\
\hline NGC 6167 & $16: 34: 36.0$ & $-49: 46: 00$ & 5.5 & 0.80 & 3.1 & $13.0 \pm 0.2$ & $\sim 2.5$ & $10.5 \pm 0.2$ & $20-30$ & - \\
\hline NGC 6193 & $16: 41: 24.0$ & $-48: 46: 00.0$ & 9.0 & 0.37 & 3.2 & $11.8 \pm 0.2$ & $\sim 1.2$ & $10.7 \pm 0.2$ & $\sim 1$ & $\sim 3-5$ \\
\hline IRAS 16375-4854 & $16: 41: 18.9$ & $-49: 00: 34.0$ & $\sim 2$ & $\sim 2.2$ & 3.4 & $\sim 18$ & $\sim 7.5$ & $\sim 10.5$ & $<1$ & - \\
\hline
\end{tabular}

Notes. Radii adopted values to select the sources for analysis in each region.
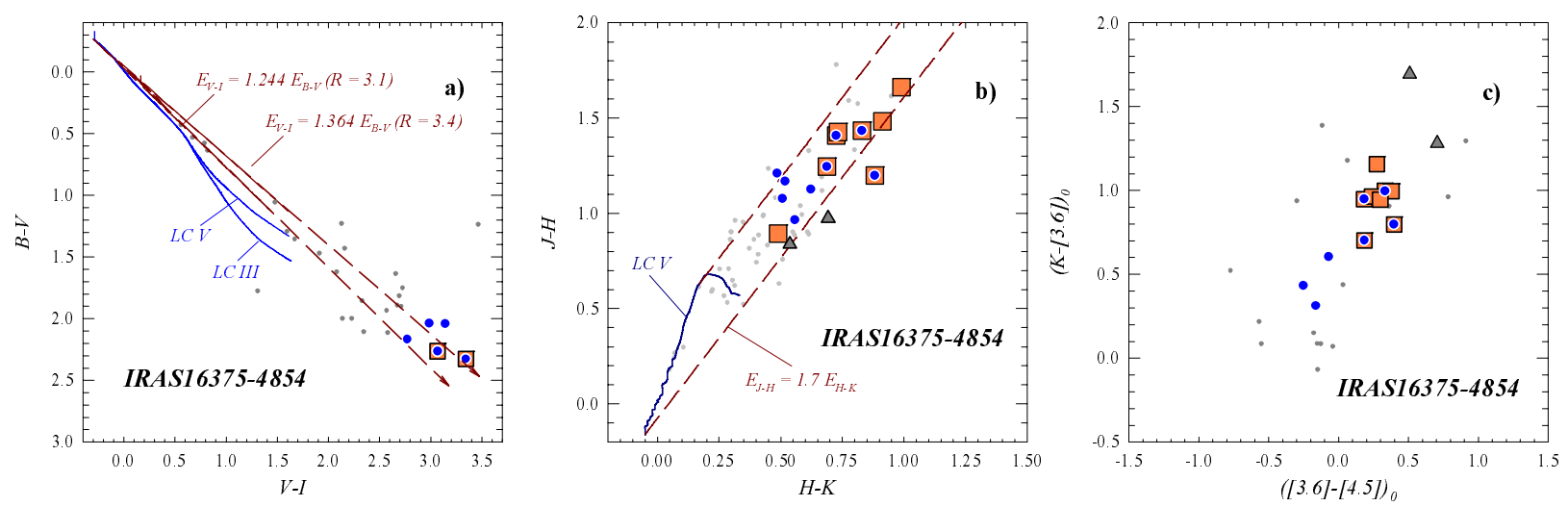

Fig. 10. NIR and MIR CCDs of stars located in IRAS 16375-4854 region. Symbols are the same as in Fig. 4. Solid curves are the intrinsic Koornneef (1983) colours for MS stars.

objects; and b) the existence of several young groups in the vicinity of NGC 6193 (see Wolk et al. 2008a,b; and Fig. 1b).

Nonetheless, in the region close to NGC 6193 there are only a few class I or class II objects, despite our identification of several likely sources as $\mathrm{H}_{\alpha}$ emitters. Low mass sources in NGC 6193 would then be somewhat older than those associated with IRAS sources and molecular clouds. In addition, the small number of class I/II sources would indicated a small, if any, age dispersion in the cluster itself (see Fig. 6c).

\subsection{Embedded clusters}

\subsubsection{IRAS 16362-4845/RCW 108-IR}

This well known IRAS source contains the remarkable embedded and massive star formation region RCW 108-IR, which has been extensively studied in several wavelength ranges (from Radio to X ray; see Arnal et al. 2005; Comerón et al. 2005; Comerón \& Schneider 2007; Wolk et al. 2008a, and references therein). Our observations cover nearly the entire region of this object. However, sources in our catalogues were selected based on their detection in the $V$ band and no further information to improve previous results could be achieved.

\subsubsection{IRAS 16375-4854 - IRAS 16379-4856}

These two IRAS sources are placed $\sim 15^{\prime}$ southward of NGC 6193 and were partially covered by our optical survey. IRAS 16379-4856 has an associated CO peak (Arnal et al. 2003) whereas IRAS 16375-4854 is located in a diffuse optical nebula that has several associated X ray sources (Wolk et al. 2008a). Our reduced IRAC SST data indicate that both IRAS sources do not seem to be independent since the objects detected in these bands form a ring structure of about $10^{\prime}$ in diameter, which is possibly a bubble centered at $\alpha_{\mathrm{J} 2000} \sim 16: 41: 37 ; \delta_{\mathrm{J} 2000} \sim-49: 02: 32$. This structure may have been generated by some energetic event at that position.

By inspecting the stars detected on both regions, we note that IRAS 16375-4854 harbors objects brighter than IRAS 163794856. By using the corresponding optical photometric diagrams (CMDs and TCDs; see Figs. 9 and 10a), we estimated its main parameters (see Table 4). They were obtained by selecting the most probable members checking simultaneously their individual position on all the photometric diagrams and also the finding chart. Apparently this region is affected by a non-ISM interstellar reddening $(R \sim 3.4)$ and has a distance similar to NGC 6193 and RCW 108-IR.

As for the infrared photometric diagrams of IRAS 163754854, the position of the selected sources as YSOs appears to be unlikely. They might be class II/III objects, although we need to analyze X-ray observations to confirm this assumption.

\section{Discussion and conclusions}

Previous investigations by our group (Vázquez et al. 1996; Baume et al. 2003) presented optical observations (typically $U B V R I$ ) of young star clusters and star forming regions. To reduce the uncertainties, we complement these UBVRI data with $\mathrm{H}_{\alpha}$ photometry, kinematic information, and near/mid infrared data. This yields more reliable values of reddening, distance, and age for NGC 6167, NGC 6193, and the IRAS 16375-4854 source. As a consequence, we can establish that all these groups are placed at the same distance from the Sun $(\sim 1300 \mathrm{pc})$ in the Sagittarius-Carina Galactic arm, but that their ages differ widely (see Table 4). We find that:

- NGC 6167 is found to be an intermediate-age cluster ( 20-30 Myr);

- NGC 6193 is very young ( 1-2 Myr) with PMS objects at different stages, $\mathrm{H}_{\alpha}$ emitters, and class II objects;

- Finally, IRAS 16375-4854 is the youngest of the three and contains several YSOs. 
In the specific case of NGC 6193, PMS stars appear to be slightly older than MS stars, i.e. the contraction age is older than the nuclear age, which might be an indication of non-coevality.

The main result of this work is the homogeneity of distances, and the differences between the ages of the Ara OB1a clusters, which may place on firmer ground future studies of the interactions between the different stellar and interstellar components of the region, such as those suggested by Arnal et al. (1987). The results presented here support the picture in which Ara OB1a is a region where star formation has proceeded for several tens of millions of years up to the present.

Acknowledgements. G.B. acknowledges ESO for granting a visitorship at Vitacura premises in March 2010, where most of this work was done. He also acknowledges the support from CONICET (PIP 5970) for the trip to CTIO on March 2006, where part of the data have been taken. We thanks very much the staff of the observatories CTIO, LCO and CASLEO during all the runs related with this work. The authors are much obliged for the use of the NASA Astrophysics Data System, of the SIMBAD database and ALADIN tools (Centre de Données Stellaires - Strasbourg, France) and of the WEBDA open cluster database. This publication also made use of data from the 2MASS, which is a project of the University of Massachusetts and the Infrared Processing and Analysis Center/California Institute of Technology, funded by the NASA and the NSF. We gratefully acknowledge use of data from the VISTA telescope, and data products from the Cambridge Astronomical Survey Unit, and support for the VVV Survey by the FONDAP Center for Astrophysics 15010003, BASAL CATA Center for Astrophysics and Associated Technologies PFB-06, MILENIO Milky Way Millennium Nucleus from the Ministry of Economy's ICM grantP07021-F, FONDECYT from CONICYT, and the European Southern Observatory. This work is based (in part) on observations made with the SST, which is operated by the JPL, California Institute of Technology under a contract with NASA. We also thank the referee, whose comments helped to improve the paper significantly.

\section{References}

Adams, M. T., Strom, K. M., \& Strom, S. E. 1983, ApJS, 53, 893

Andre, P., Ward-Thompson, D., \& Barsony, M. 1993, ApJ, 406, 122

Arnal, E. M., Cersosimo, J. C., May, J., \& Bronfman, L. 1987, A\&A, 174, 78

Arnal, E. M., May, J., \& Romero, G. A. 2003, A\&A, 412, 431

Arnal, E. M., Romero, G. A., May, J., \& Minniti, D. 2005, ASPC, 344, 173

Baume, G., Vázquez, R. A., Carraro, G., \& Feinstein, A. 2003, A\&A, 402, 549

Baume, G., Moitinho, A., Giorgi, E. E., Carraro, G., \& Vázquez, R. A. 2004, A\&A, 417, 961

Baume, G., Moitinho, A., Vázquez, R. A., et al. 2006, MNRAS, 367, 1441

Baume, G., Carraro, G., \& Momany, Y. 2009, MNRAS, 398, 221

Bessell, M. S., \& Brett, J. M. 1988, PASP, 100, 1134

Bruck, M. T., \& Smyth, M. J. 1967, MNRAS, 136, 431

Cabrero-Caño, J., \& Alfaro, E. J. 1985, A\&A, 150, 298

Carraro, G., \& Munari, U. 2004, MNRAS, 347, 625
Carraro, G., Baume, G., Piotto, G., Méndez, R. A., \& Schmidtobreick, L. 2005, A\&A, 436, 527

Comerón, F., \& Schneider, N. 2007, A\&A, 473, 149

Comerón, F., Schneider, N., \& Russeil, D. 2005, A\&A, 433, 955

Cousins, A. W. J. 1978a, MNSSA, 37, 62

Cousins, A. W. J. 1978b, MNSSA, 37, 77

Cutri, R. M., Skrutskie, M. F., Van Dyk, S., et al. 2003, University of Massachusetts and Infrared Processing and Analysis Center (California Institute of Technology)

Dias, W. S., Alessi, B. S., Moitinho, A., et al. 2002, A\&A, 389, 871

Dias, W. S., Assafin, M., Flório, V., Alessi, B. S., \& Líbero, V. 2006, A\&A, 446, 949

Didelon, P. 1982, A\&AS, 50, 199D

Feigelson, E. D., \& Kriss, G. A. 1983, AJ, 88, 431

Fitzgerald, M. P. 1987, MNRAS, 229, 227

Flaherty, K. M., Pipher, J. L., Megeath, S. T., et al. 2007, ApJ, 663, 1069

Gutermuth, R. A., Myers, P. C., Megeath, S. T., et al. 2008, ApJ, 674, 336

Kaltcheva, N. T., \& Georgiev, L. N. 1992, MNRAS, 259, 166

Kenyon, S. J., \& Hartmann, L. W. 1990, ApJ, 349, 197

Koornneef, J. 1983, A\&A, 128, 84

Lada, C. J. 1987, in Star Forming Regions, ed. M. Peimbert, \& J. Jugaku (Dordrecht: Reidel), IAU Symp., 115, 1

Landolt, A. U. 1992, AJ, 104, 340

Marigo, P., Girardi, L., Bressan, A., et al. 2008, A\&A, 482, 883

Minniti, D., Lucas, P. W., Emerson, J. P., et al. 2010, NewA, 15, 433

Moffat, A. F. J., \& Vogt, N. 1973, A\&AS, 10, 135

Moffat, A. F. J., \& Vogt, N. 1975, A\&AS, 20, 155

Neckel, Th., \& Klare, G. 1980, A\&AS, 42, 251

Niemela, V. S., \& Gamen, R. C. 2005, MNRAS, 356, 974

Preibisch, T., \& Zinnecker, H. 1999, AJ, 117, 2381

Rizzo, J. R., \& Bajaja, E. 1994, A\&A, 289, 922

Schmidt-Kaler, Th. 1982, Landolt-Börnstein, Numerical data and Functional Relationships in Science and Technology, New Series, Group VI, Vol. 2(b), ed. K. Schaifers, \& H. H. Voigt (Berlin: Springer Verlag), 14

Stetson, P. B. 1987, PASP, 99, 191

Stetson, P. B. 1992, in Stellar Photometry-Current Techniques and Future Developments, ed. C. J. Bulter, \& I. Elliot (Cambridge: Cambridge University Press), IAU Coll., 136, 291

Skrutskie, M. F., Cutri, R. M., Stiening, R., et al. 2006, AJ, 131, 1163

Siess, L., Dufour, E., \& Forestini, M. 2000, A\&A, 358, 593

Sugitani, K., \& Ogura, K. 1994, ApJS, 92, 163

Sugitani, K., Fukui, Y., \& Ogura, K. 1991, ApJS, 77, 59

Sung, H., Bessell, M. S., \& Lee, S.-W. 1998, AJ, 115, 734

Urquhart, J. S., Morgan, L. K., \& Thompson, M. A. 2009, A\&A, 497, 789

Vázquez, R. A., \& Feinstein, A. 1992, A\&AS, 92, 863

Vázquez, R. A., Baume, G., Feinstein, A., \& Prado, P. 1996, A\&AS, 116, 75

Waldhausen, S., Martínez, R. E., \& Feinstein, C. 1999, AJ, 117, 2882

Wolk, S. J., Spitzbart, B. D., Bourke, T. L., et al. 2008a, AJ, 135, 693

Wolk, S. J., Comerón, F., \& Bourke, T. 2008b, in Handbook of Star Forming Regions, Vol. II: The Southern Sky ASP Monograph Publications, ed. B. Reipurth, 5, 388

Zacharias, N., Finch, C., Girard, T., et al. 2010, AJ, 139, 2184

Zhao, J. L., \& He, Y. P. 1990, A\&A, 237, 54 